\title{
A Dynamic Substrate is Required for MhuD-catalysed Degradation of Haem to Mycobilin
}

Received 00th January 20xx, Accepted 00th January 20xx DOI: $10.1039 / \times 0 \times x 00000 x$

\author{
Biswash Thakuri, Bruce D. O’Rourke, Amanda B. Graves, and Matthew D. Liptak*
}

\begin{abstract}
The non-canoncial haem oxygenase MhuD from Mycobacterium tuberculosis binds a haem substrate that adopts a dynamic equilibrium between planar and out-of-plane ruffled conformations. MhuD degrades this substrate to an unusual mycobilin product via successive monooxygenation and dioxygenation reactions. This article establishes a causal relationship between haem substrate dynamics and MhuD-catalysed haem degradation resulting in a revised enzymatic mechanism. UV/Vis absorption (Abs) and electrospray ionisation mass spectrometry (ESI-MS) data demonstrated that a second-sphere substitution favouring population of the ruffled haem conformation changed the rate-limiting step of the reaction resulting in a measurable build-up of the monooxygenated meso-hydroxyhaem intermediate. In addition, UV/Vis Abs and ESI-MS data for a second-sphere variant that favoured the planar substrate conformation showed that this change altered the enzymatic mechanism resulting in an $\alpha$-biliverdin product. Single-turnover kinetic analyses for three MhuD variants revealed that the rate of haem monooxygenation depends upon the population of the ruffled substrate conformation. These kinetic analyses also revealed that the rate of meso-hydroxyhaem dioxygenation by MhuD depends upon the population of the planar substrate conformation. Thus, the ruffled haem conformation supports rapid haem monooxygenation by MhuD, but further oxygenation to the mycobilin product is inhibited. In contrast, the planar substrate conformation exhibits altered haem monooxygenation regiospecificity followed by rapid oxygenation of meso-hydroxyhaem. Altogether, these data yielded a revised enzymatic mechanism for MhuD where access to both substrate conformations is needed for rapid incorporation of three oxygen atoms into haem yielding mycobilin.
\end{abstract}

\section{Introduction}

At physiologically-relevant temperatures, proteins vibrate around their equilibrium structure and these dynamic motions are often essential for their function. Sometimes dynamic motion is dramatic, as is the case for the domain rearrangements of cobalamin-dependent methionine synthase during enzymatic turnover. ${ }^{1-3}$ Other times, more subtle structural changes have important functions as is the case with the allosteric changes to haemoglobin following binding of molecular oxygen. ${ }^{4-6}$ In haem proteins, a source of dynamic motion is out-of-plane deformations of the haem substrate. ${ }^{7-9}$ For clarity, these motions are described based upon the normal coordinates for distortion from a $D_{4 h}$ symmetric tetrapyrrole: waving $\left(e_{g}\right)$, propellering $\left(a_{1 u}\right)$, doming $\left(a_{2 u}\right)$, ruffling $\left(b_{1 u}\right)$, and saddling $\left(b_{2 u}\right)$. A prominent example of a protein that hosts a dynamic haem is Mycobacterium tuberculosis MhuD. ${ }^{10-12}$ MhuD is a non-canonical haem oxygenase $(\mathrm{HO})$ whose haem substrate undergoes a dynamic ruffling deformation, but it has not yet

Department of Chemistry, University of Vermont, Burlington, Vermont 05405 , USA.

E-mail: matthew.liptak@uvm.edu

+Electronic Supplementary Information (ESI) available: FPLC traces, SDS-PAGE gels, MS data, and complete derivations of equations 1 and 2 .

See DOI: $10.1039 / x 0 x x 00000 x$ been ascertained whether the substrate dynamics have a functional role.

It has been shown that MhuD binds a dynamic haem and that MhuD degrades haem to a unique product, but a connection between haem ruffling and mycobilin production has been elusive. Magnetic measurements have shown that the large ruffling deformation observed in the X-ray crystal structure of cyanide-inhibited MhuD (MhuD-haem-CN, PDB ID $4 \mathrm{NL5}$ ) arises from a dynamic haem substrate that is present in solution as an equilibrium mixture of planar and ruffled haem. ${ }^{10-}$ 12 One explanation for the presence of two haem conformations in MhuD is that it is a consequence of the fact that at least four polypeptide conformational states exist. ${ }^{10,13,14}$ Alternatively, from a haem electronic structure viewpoint, the ruffled conformation can be attributed to a hidden pseudo Jahn-Teller distortion arising from vibronic coupling of the ${ }^{2} B_{2 g}$ and ${ }^{2} A_{2 u}$ excited states of the planar species by a $b_{1 u}$ vibration. ${ }^{12,15,16}$ The populations of these conformations can be tuned by the $\mathrm{F} 23 \mathrm{~W}$ and $\mathrm{W} 66 \mathrm{~F}$ substitutions because these residues act like a vice on the haem substrate (Fig. 1). ${ }^{11}$ As alluded to above, MhuD is a non-canonical $\mathrm{HO}$ that degrades haem to mycobilin via a mesohydroxyhaem intermediate. ${ }^{17,}{ }^{18}$ Canonical HOs, which degrade haem to biliverdin, also have a meso-hydroxyhaem intermediate, suggesting the mechanisms of the two enzymes diverge after the first oxygenation reaction. ${ }^{19}$ These observations suggest that haem ruffling induced by the MhuD active site may be responsible for the novel mycobilin product 

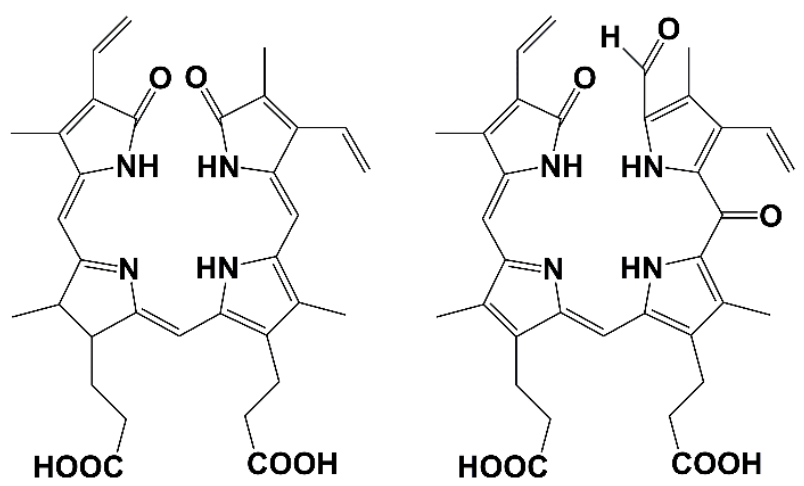

Biliverdin

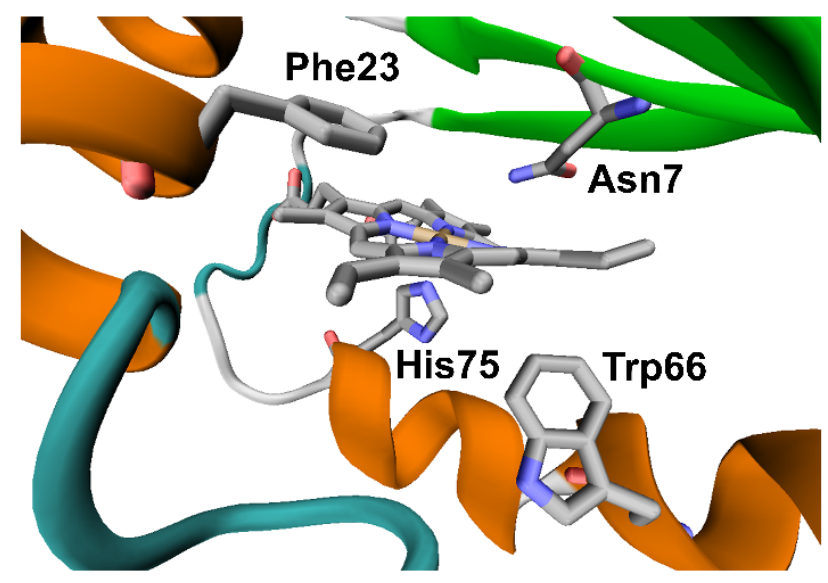

Fig. 1 MhuD is a non-canonical haem oxygenase whose haem degradation products and active site structure differ from canonical enzymes. MhuD produces a trioxygenated mycobilin product, whereas canonical haem oxygenases generate dioxygenated biliverdin (top). His75 is a first-sphere ligand to the haem substrate. Asn7, Phe23, and Trp66 are second-sphere ligands that perturb the haem substrate via non-bonding interactions (bottom).

of this enzyme, but no clear correlation between haem ruffling and $\mathrm{HO}$ degradation product has been reported.

To assess the role(s) of a dynamic haem in the MhuD enzyme mechanism, we identified the isomeric products and measured rate constants for MhuD-catalysed haem oxygenation as a function of substrate conformation. We determined the isomeric products of MhuD-catalysed haem degradation for enzyme variants that stabilize one of two substrate conformations. ${ }^{12}$ Wild-type (WT) MhuD is known to produce mycobilin, and the R26S variant has been shown to generate predominantly $\alpha$-biliverdin, ${ }^{20}$ but the origin of this difference had yet to be explained. The Michaelis-Menten model is problematic for MhuD-catalysed haem oxygenation because: MhuD binds the haem substrate tightly with a $K_{\mathrm{d}}$ of 7.6 $\mathrm{nM},{ }^{21}$ MhuD-catalysed haem oxygenation is a multi-step reaction, ${ }^{18}$ and the enzyme is product-inhibited in vitro. ${ }^{14}$ Thus, we have derived single-turnover kinetic expressions that are appropriate for the MhuD-catalysed reaction in this article. The enzyme catalysed conversion of haem to meso-hydroxyhaem is formally a monooxygenation, ${ }^{18}$ and the influence of ruffling on the rate of this reaction has been assessed using an established assay. ${ }^{13}$ The further oxygenation of meso-hydroxyhaem to mycobilin is formally a dioxygenation reaction, ${ }^{17}$ and the rate of this reaction was assessed here by a novel enzyme assay. These experiments have provided important insight into the role of dynamic haem ruffling in the enzymatic mechanism of MhuD.

This article describes a mechanistic study of MhuDcatalysed haem oxygenation carried out using UV/Vis absorption (Abs) spectroscopy and mass spectrometry (MS). Forms of MhuD with increased populations of the ruffled and planar substrate conformations were prepared by introducing the F23W and W66F substitutions, ${ }^{11,12}$ respectively. The haem degradation products of WT, F23W, and W66F MhuD were identified using

UV/Vis Abs spectroscopy and in proteo MS. ${ }^{22}$ In addition, tandem MS was employed to determine the product isomer(s) for each reaction. Next, using a previously described UV/Vis Abs-based assay, ${ }^{13}$ the rates of WT, F23W, and W66F MhuDcatalysed haem monooxygenation were measured by monitoring the disappearance of the Soret band. Finally, the rate of meso-hydroxyhaem oxygenation was quantified by analysing the time course of a UV/Vis Abs band in the red region of the spectrum. These data have provided significant new insight into the enzymatic mechanism of MhuD, and revealed a new example of protein function derived from protein dynamics.

\section{Experimental}

All reagents were purchased from Fisher Scientific and used without further purification unless noted otherwise.

\section{Protein expression and purification}

WT, F23W, and W66F MhuD were expressed as described previously. Briefly, a pET-22b (Amp ${ }^{r}$ ) plasmid encoding WT MhuD was a gift from Prof. Celia Goulding (University of California-Irvine). ${ }^{13}$ The F23W and W66F mutations were introduced using QuikChange site-directed mutagenesis kits (Agilent). ${ }^{11,12}$ All three MhuD variants were overexpressed in BL21-GOLD (DE3) cells, grown aerobically in Luria-Bertani (LB) medium, as described previously.

WT, F23W, and W66F MhuD were purified in a manner similar to that previously described for $\mathrm{His}_{6}$-tagged WT enzyme. WT MhuD was purified using an ÄKTA pure $25 \mathrm{~L}$ fast protein liquid chromatography (FPLC) system. ${ }^{21}$ F23W MhuD was purified similarly, but a $1.3 \mathrm{mM} / \mathrm{mL}$ elution gradient was employed (Fig. S1). This procedure yielded $18 \mathrm{mg}$ of $>99 \%$ pure F23W MhuD per liter of LB medium (Fig. S3). W66F MhuD was also purified similarly to WT enzyme, but with an elution gradient of $3.3 \mathrm{mM} / \mathrm{mL}$ from 125 to $325 \mathrm{mM}$ imidazole (Fig. S3). Purification of W66F MhuD yielded $15 \mathrm{mg}$ of $>99 \%$ pure protein per liter of growth medium, as assessed by SDS-PAGE (Fig. S4).

\section{UV/Vis Abs spectroscopy}

Haem degradation by MhuD variants was assayed in a manner similar to previously described experiments. WT, F23W, and W66F haem-bound MhuD (MhuD-haem) were prepared as previously described. ${ }^{10}, 13 \mathrm{UV} / \mathrm{Vis}$-detected haem degradation 
assays were performed in a manner similar to that previously described, ${ }^{17}$ with several exceptions. Briefly, $50 \mu \mathrm{M}$ MhuDhaem in $50 \mathrm{mM}$ potassium phosphate $\left(\mathrm{KP}_{\mathrm{i}}\right) \mathrm{pH} 6.0$ was reacted with $5 \mathrm{mM}$ ascorbic acid, $10 \mathrm{mM}$ ethylenediaminetetraacetic acid (EDTA), $840 \mathrm{U} / \mathrm{mL}$ bovine catalase (Sigma-Aldrich), and 167 $\mathrm{U} / \mathrm{mL}$ bovine superoxide dismutase at $37{ }^{\circ} \mathrm{C}$ while protected from light. For each enzyme variant, UV/Vis Abs spectra were acquired between 700 and $300 \mathrm{~nm}$ with a scan rate of 600 $\mathrm{nm} / \mathrm{min}$, a $1.0 \mathrm{~nm}$ data interval, and a $0.1 \mathrm{~s}$ integration time using a Cary 100 Bio UV-Vis spectrophotometer at 0, 5, 10, 20, 30,60 , and 120 min after mixing.

Haem degradation by F23W MhuD was also monitored at a lower temperature with higher time resolution to gain further insight into the reaction catalysed by this enzyme variant. 20 $\mu \mathrm{M}$ F23W MhuD-haem in $50 \mathrm{mM} \mathrm{KP}$ i $\mathrm{pH} 6.0$ was reacted with 2 $\mathrm{mM}$ ascorbic acid, $5 \mathrm{mM}$ EDTA, $840 \mathrm{U} / \mathrm{mL}$ bovine catalase, and $167 \mathrm{U} / \mathrm{mL}$ bovine superoxide dismutase at room temperature while protected from light. A UV/Vis Abs spectrum of the reaction mixture was acquired every $60 \mathrm{~s}$ for $120 \mathrm{~min}$ using the same instrumental parameters noted above.

\section{Mass Spectrometry}

Electrospray ionisation mass spectrometry (ESI-MS) was used to monitor haem degradation by WT, F23W, and W66F MhuD as a function of time. WT, F23W, and W66F MhuD samples with partially degraded haem were prepared as described above and flash frozen in liquid nitrogen (Airgas) after $0,5,10,20,30,60$, and 120 min of reaction time. In addition, a control experiment was performed where $50 \mu \mathrm{M}$ hemin chloride in $50 \mathrm{mM} \mathrm{KPi}$ pH 6.0 was reacted with $5 \mathrm{mM}$ ascorbic acid, $10 \mathrm{mM}$ EDTA, $840 \mathrm{U} / \mathrm{mL}$ bovine catalase (Sigma-Aldrich), and $167 \mathrm{U} / \mathrm{mL}$ bovine superoxide dismutase at $37^{\circ} \mathrm{C}$ for $120 \mathrm{~min}$ before flash freezing the sample in liquid nitrogen. All 22 reactions were loaded onto a $\mathrm{C}_{18}$ column equilibrated with $2 \%$ acetonitrile (MeCN) with $0.1 \%$ formic acid $(v / v)$ in water $(v / v)$, and eluted with a linear gradient from $2 \%$ to $98 \% \mathrm{MeCN}$ with $0.1 \%$ formic acid $(v / v)$ in water $(v / v)$ over 50 min using a Shimadzu Prominence high-performance liquid chromatography (HPLC) system. ESI-MS data for m/z 600-1500 was acquired in positive ion mode using an ABI Sciex 4000 QTrap Pro hybrid triple-quadrupole/linear ion trap. Extracted ion chromatograms (EICs) for $\mathrm{m} / \mathrm{z} 583,611$, and 616 target ions were obtained from each total ion chromatogram using BioAnalyst 1.5. Next, each EIC was background subtracted and the integrated areas of each ion as a function of time were determined by integrating under the corresponding chromatographic peak in the background subtracted EIC. The integrated areas of the $\mathrm{m} / \mathrm{z} 583$ and 611 target ions relative to the $\mathrm{m} / \mathrm{z} 616$ target ion at each time point were plotted against time.

Tandem LC-MS experiments were employed to identify the major ions produced by the WT, F23W, and W66F MhuDcatalysed reactions. Tandem LC-MS data were obtained for the $\mathrm{m} / \mathrm{z} 583,611$ and 616 parent ions of the WT, W66F, and F23W MhuD-catalysed haem degradation reactions. Evidence for several m/z 583 isomers was observed, so the 120 minute reactions samples were further separated by liquid chromatography. Up to four isomers were resolved by a linear gradient from $2 \%$ to $82 \% \mathrm{MeCN}$ with $0.1 \%$ formic acid $(v / v)$ in water $(v / v)$ over $38 \mathrm{~min}$. All Tandem LC-MS data were acquired using the same instrument described above for ESI-MS. A collision energy of $44 \mathrm{mV}$ was used for fragmentation and the third quadrupole was used as a linear ion trap to scan the fragmentation products of the parent ions.

\section{Kinetic Models}

Kinetic information was extracted from the UV/Vis Abs data via non-linear least squares fitting to single-turnover kinetic models. The decrease of the MhuD-haem Soret band as a function of time was fit to a pseudo-first order kinetic model:

$A_{\text {Soret }}=\left(A_{\text {Soret }, 0}-A_{\text {Soret }, \infty}\right) e^{-k_{1} t}+A_{\text {Soret }, \infty}$

where $A_{\text {soret, } 0}$ is the absorbance at $0 \mathrm{~min}, A_{\text {soret, } \infty}$ is the absorbance at $\infty \mathrm{min}$, and $k_{1}$ is the rate constant for haem monooxygenation. The decrease of the Soret band Abs intensity versus time was fit to equation 1 using GraphPad Prism 8.0 in order to extract $k_{1}$ and its standard error for each MhuD variant.

For F23W MhuD, the time-course of $620 \mathrm{~nm}$ UV/Vis Abs feature was described by the following kinetic model:

$A_{620}=A_{620,0}\left[e^{-k_{1} t}+\left(\frac{\varepsilon_{m h}}{\varepsilon_{h}}\right)\left(\frac{k_{1}}{k_{2}-k_{1}}\right)\left(e^{-k_{1} t}-e^{-k_{2} t}\right)\right]$

where $A_{620,0}$ is the absorbance at $0 \mathrm{~min}, k_{1}$ is the rate constant for F23W MhuD-catalysed haem monooxygenation determined from fitting the Soret band decrease to equation $1, \varepsilon_{m h}$ is the molar extinction coefficient for meso-hydroxyhaem at $620 \mathrm{~nm}$, $\varepsilon_{h}$ is the molar extinction coefficient for haem at $620 \mathrm{~nm}$, and $k_{2}$ is the rate constant for haem dioxygenation. $\varepsilon_{\mathrm{h}}$ was determined based upon the pyridine hemochrome assay. ${ }^{23}$ The change in absorbance at $620 \mathrm{~nm}$ versus time was fit to equation 2 using GraphPad Prism 8.0 to extract $k_{2}, \varepsilon_{m h}$, and their standard errors. Complete derivations of equations 1 and 2 are available in the ESI.

\section{Results}

\section{The MhuD product depends upon the substrate conformation}

Based upon UV/Vis Abs data, WT MhuD degraded haem to mycobilin. As reported previously, two substrate conformations that differ by their degree of out-of-plane ruffling are in dynamic equilibrium within the active site of WT enzyme. ${ }^{12}$ Similar to previously reported assays, ${ }^{13}, 17$ a reaction was initiated by adding a 100 -fold molar excess of ascorbate to 50 $\mu \mathrm{M}$ MhuD-haem in $50 \mathrm{mM} \mathrm{KP}$ pH 6.0 at $37^{\circ} \mathrm{C}$ and the UV/Vis Abs spectrum of the mixture was monitored versus time (Fig. 2). ${ }^{13,17}$ It should be noted that ascorbate is unlikely to be the physiological electron donor for this reaction, and the redox partner for MhuD has yet to be identified. As was also the case for previous studies that employed cytochrome $P_{450}$ reductase as the electron donor, ${ }^{13}$ or substituted hydrogen peroxide for both molecular oxygen and the electron donor, ${ }^{18}$ the reaction significantly decreased the Soret band intensity and produced a 
new UV/Vis Abs band at $540 \mathrm{~nm}$. Based upon the UV/Vis Abs spectrum of mycobilin-bound MhuD, ${ }^{18}$ these observations are

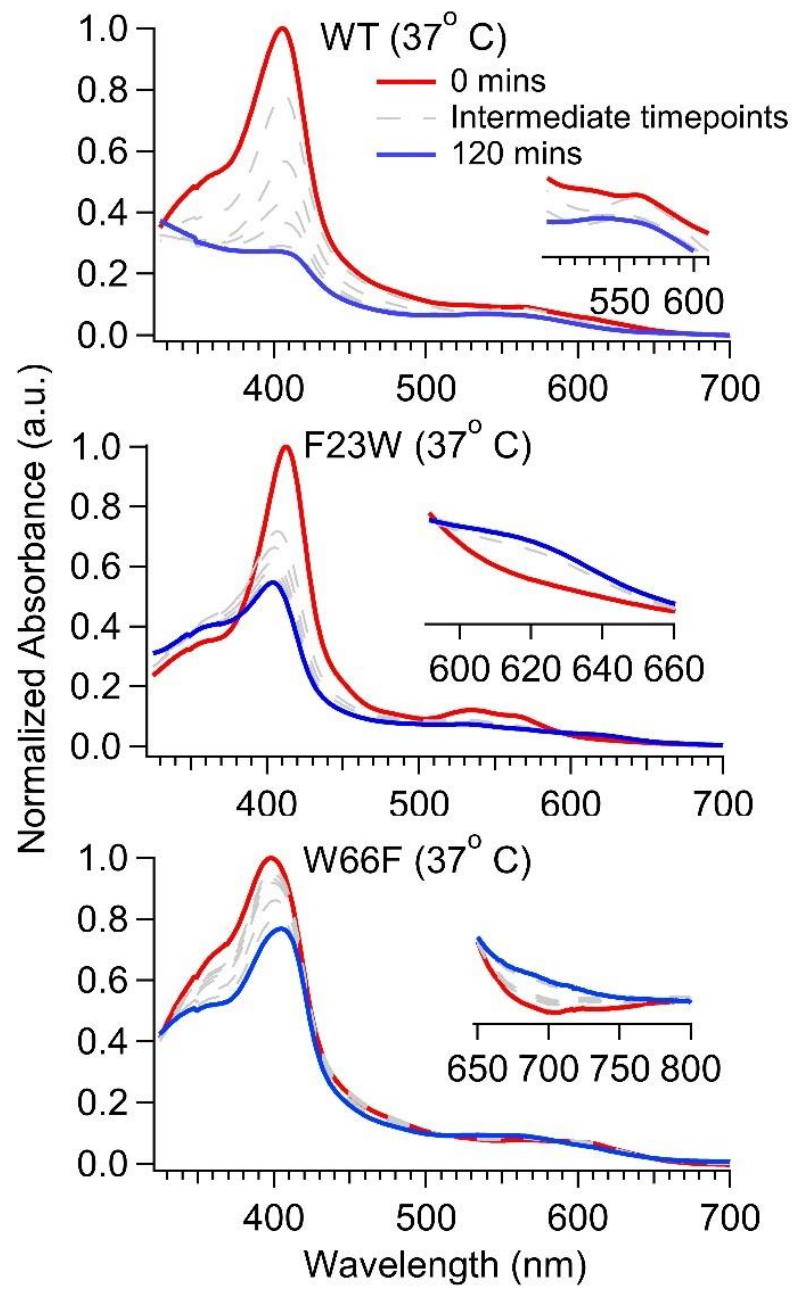

Fig. 2 Second-sphere substitutions alter the enzymatic product of MhuD. WT MhuD degrades haem to a new species with a UV/Vis Abs band at $540 \mathrm{~nm}$ (top), which is consistent with production of mycobilin. F23W MhuD-cataylsed haem degradation yields a species with a UV/Vis Abs band at $620 \mathrm{~nm}$ (middle), consistent with a build-up of the meso-hydroxyhaem intermediate. Haem degradation by W66F MhuD results in a product with a broad UV/Vis Abs band at $680 \mathrm{~nm}$, which suggests biliverdin production. For each variant, the reaction of $50 \mu \mathrm{M}$ MhuD-haem with $5 \mathrm{mM}$ ascorbate in $50 \mathrm{mM} \mathrm{KP}$ $\mathrm{pH} 6.0$ was monited by UV/Vis Abs spectroscopy for $2 \mathrm{~h}$ at $37^{\circ} \mathrm{C}$

consistent with oxygenation of haem to mycobilin by WT enzyme with ascorbate as the electron donor under aerobic conditions. Thus, this study has successfully reproduced past observations regarding the haem degradation reaction catalysed by WT MhuD, and the role of the two substrate conformations can now be examined.

When the ruffled substrate conformation was favoured by the F23W substitution, the UV/Vis Abs spectrum of the reaction mixture could be attributed to a build-up of the mesohydroxyhaem intermediate. A previous study demonstrated that the F23W substitution favours population of the ruffled substrate conformation by increasing steric contacts between residue 23 and the porphyrin ring. ${ }^{12}$ The reaction of F23W MhuD with excess ascorbate was monitored by UV/Vis Abs as described above for the WT form of the enzyme, and the formation of a novel $620 \mathrm{~nm}$ band was observed (Fig. 2). Based upon the UV/Vis Abs spectrum of meso-hydroxyhaem-bound MhuD, and what is known regarding the mechanisms of WT enzyme, we concluded that the UV/Vis Abs band corresponds to a build-up of the meso-hydroxyhaem intermediate. ${ }^{18}$ In addition, the UV/Vis Abs spectrum acquired following $2 \mathrm{~h}$ of haem degradation by F23W MhuD has a significantly more intense absorption in the $350-450 \mathrm{~nm}$ region compared to the corresponding data for WT enzyme. This observation is also consistent with formation of meso-hydroxyhaem by F23W MhuD since that species retains a Soret band. These data strongly suggest that F23W MhuD has the ability to monooxygenate haem, but subsequent oxygenation reactions may be impaired in this variant.

When the planar substrate conformation was favoured by the W66F substitution, an alternate haem degradation product was generated whose UV/Vis Abs spectrum was consistent with biliverdin. The W66F substitution was previously shown to favour population of the planar substrate conformation by reducing a steric clash between residue 66 and the porphyrin ring. ${ }^{11}$ W66F MhuD was assayed under identical conditions to WT enzyme and, surprisingly, production of a broad $680 \mathrm{~nm}$ UV/Vis Abs band over the course of haem degradation reaction was noted (Fig. 2). Based upon the UV/Vis Abs spectrum of biliverdin-bound rat $\mathrm{HO}^{24}$ a reasonable assignment for this band is biliverdin. Biliverdin production has been reported previously for R26S MhuD, ${ }^{20}$ but the mechanistic changes that lead to this alternate product remain unknown. The data presented here suggests that the planar substrate conformation may play a role in biliverdin production by MhuD. However, first, the observation that W66F MhuD may degrade haem to biliverdin will be further investigated with MS below.

\section{The major product of the ruffled conformation is mycobilin}

Surprisingly, ESI-MS data for the reaction mixtures revealed that WT, F23W, and W66F MhuD degraded haem to a mixture of products. WT, F23W, and W66F MhuD-haem were allowed to react with a 100 -fold excess of ascorbate for $30 \mathrm{~min}$ at $37^{\circ} \mathrm{C}$, as described above for the UV/Vis Abs-detected assays. In contrast to previous studies that have isolated MhuD degradation products prior to MS analysis, ${ }^{17,20}$ here the crude reaction mixture was analysed by in proteo LC-MS. ${ }^{22}$ LC-MS of the WT and F23W MhuD reaction mixtures revealed a major product with $\mathrm{m} / \mathrm{z} 611$ and a minor product with $\mathrm{m} / \mathrm{z} 583$ (Fig. 3). In contrast, the major product ion for the W66F MhuDcatalysed reaction had a molecular weight of $583 \mathrm{Da}$ and the 611 Da species was the minor product. As a control, the same reaction was carried out in the absence of enzyme yielding small amount of the 583 Da product and no detectable production of the 611 Da product (Fig. S5). Thus, MhuD degrades haem to two products, with molecular weights of 611 and $583 \mathrm{Da}$, and the W66F substitution changes the identity of the major product.

Tandem LC-MS was employed to identify the 611 and 583 Da products of MhuD-catalysed haem degradation as mycobilin and biliverdin, respectively. The tandem LC-MS fragmentation 
pattern for $\mathrm{m} / \mathrm{z} 616$ ion is very similar to that of authentic haem (Fig. S6), identifying this species as unreacted substrate. Tandem LC-MS of the 611 Da product ion for WT enzyme

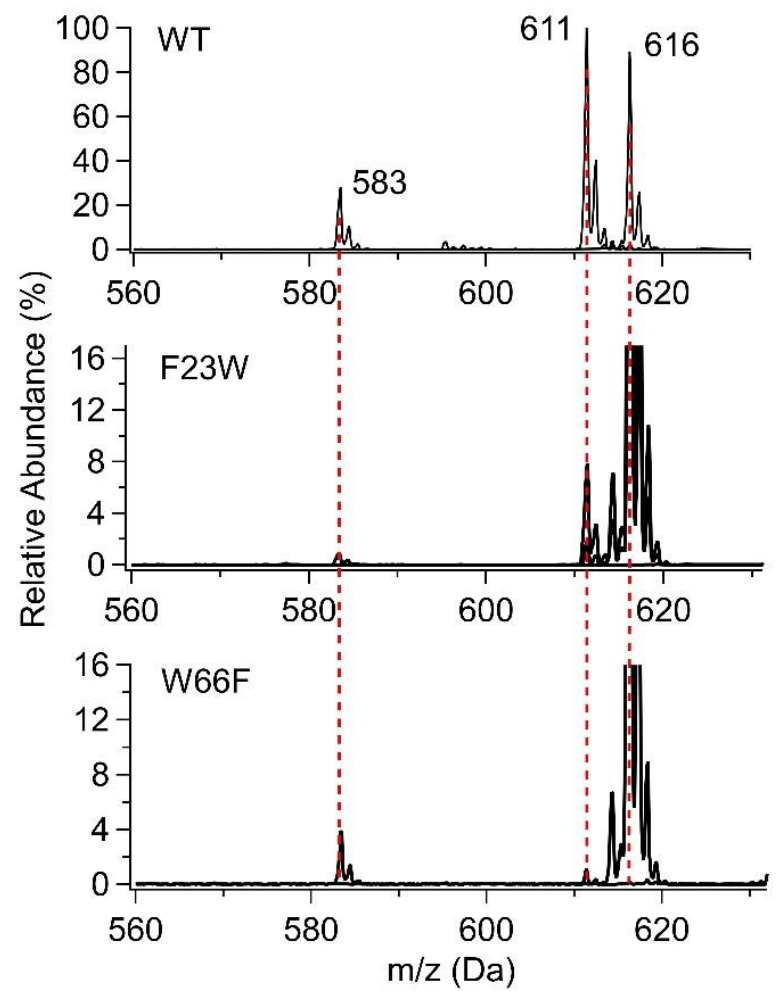

Fig. 3 ESI-MS reveals that MhuD degrades haem to a mixture of mycobilin and biliverdin, and the ratio depends upon second-sphere interactions within the enzyme active site. WT MhuD degrades haem $(\mathrm{m} / \mathrm{z} 616)$ to a major mycobilin product $(\mathrm{m} / \mathrm{z} 611)$ and minor biliverdin product ( $\mathrm{m} / \mathrm{z} 583$, top). The major product of F23W MhuD is also mycobilin, but enzymatic turnover is severely impaired (middle). In contrast, the major product of W66F MhuD is biliverdin (bottom). ESI-MS data were acquired after $50 \mu \mathrm{M}$ MhuD-haem was permitted to react with $5 \mathrm{mM}$ ascorbate in $50 \mathrm{mM} \mathrm{KP}$ i $\mathrm{pH} 6.0$ for $30 \mathrm{~min}$ at $37^{\circ} \mathrm{C}$

yielded a major fragment ion at $\mathrm{m} / \mathrm{z} 476$ along with severa minor fragments (Fig. S7). The $476 \mathrm{Da}$ fragment ion corresponds to fragmentation of mycobilin- $a$ between the $\delta$-meso carbon and pyrrole ring $D$, or fragmentation of mycobilin- $b$ between the $\beta$-meso carbon and pyrrole ring $C$. The minor fragment ions can also be assigned to reasonable fragmentations of mycobilin. Finally, tandem LC-MS was also performed for the $583 \mathrm{Da}$ product, yielding a single fragment ion at $\mathrm{m} / \mathrm{z} 297$ (Fig. S8). This fragmentation was previously assigned to a biliverdin product of R26S MhuD-catalysed haem degradation. ${ }^{20}$ Based upon these data, we can conclude that MhuD produces a mixture of mycobilin and biliverdin products.

Mycobilin and biliverdin production by WT, F23W, and W66F MhuD was monitored by ESI-MS to ascertain the influence of substrate conformation on product identity. The WT, F23W, and W66F MhuD reaction mixtures described above were flash-frozen at seven time points ranging from 0 to 120 min, and the product distributions were analysed by ESI-MS (Fig. 4). Starting from the $20 \mathrm{~min}$ time point, the major product of WT MhuD is clearly mycobilin in accord with a previous study that relied upon product extraction. ${ }^{17}$ Prior to the 20 minute time point, mycobilin and biliverdin production by WT enzyme appears similar, but it is difficult to say whether this observation is due to different rates for the two haem degradation reactions or different ionisation efficiencies for mycobilin and biliverdin.
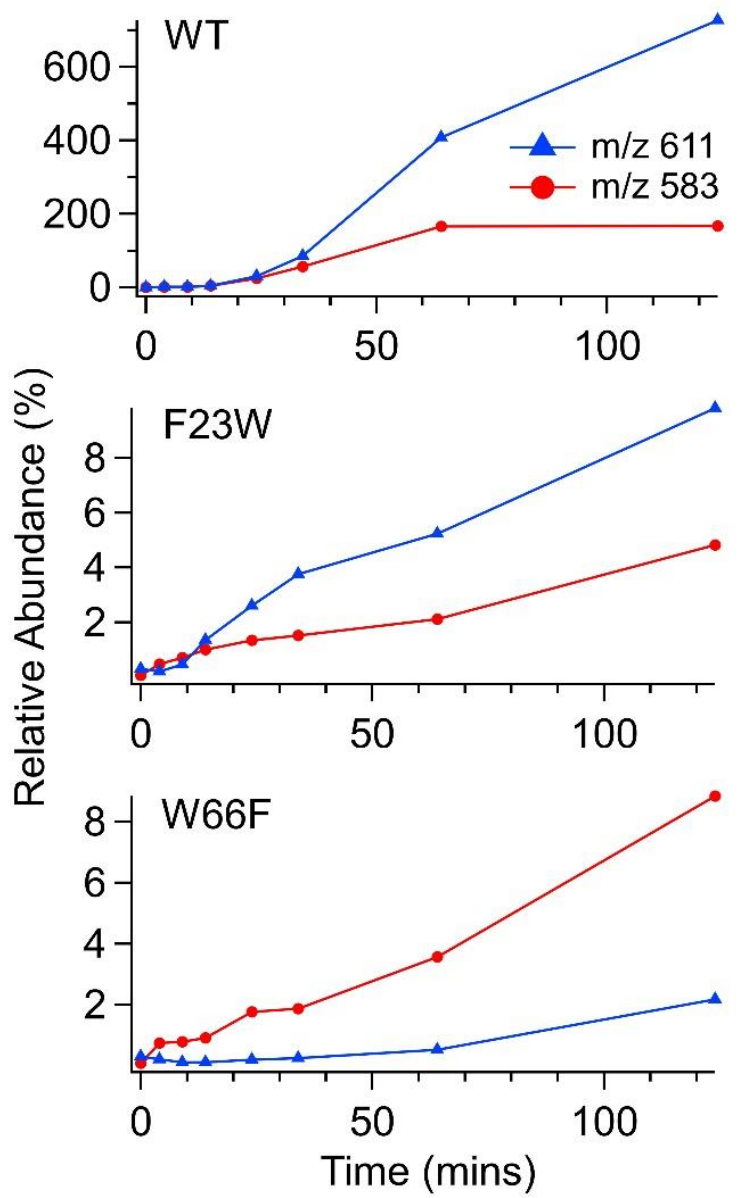

Fig. 4 The production of mycobilin $(\mathrm{m} / \mathrm{z} 611)$ and biliverdin $(\mathrm{m} / \mathrm{z} 583)$ was monitored as a function of time for three variants of MhuD with altered second-sphere interactions. WT MhuD consistently produces mycobilin as a major product over the course of $2 \mathrm{~h}$ (top). F23W MhuD initially produces a larger fraction of biliverdin, but mycobilin production predominates after $20 \mathrm{~min}$ (middle). The major product of W66F MhuD is biliverdin (bottom). ESI-MS data were acquired for aliquots of a $37^{\circ} \mathrm{C}$ reaction between $50 \mu \mathrm{M}$ MhuD-haem and $5 \mathrm{mM}$ ascorbate in $50 \mathrm{mM} \mathrm{KP}, \mathrm{pH} 6.0$ that were flash-frozen after $0,5,10,20,30,60$, and $120 \mathrm{~min}$. Mycobilin and biliverdin abundances are reported relative to the haem abundance at each time point.

When population of the ruffled conformation was favoured by the F23W substitution, mycobilin remained the major product but the rate of enzymatic turnover decreased dramatically. Finally, when population of the planar conformation was favoured by the W66F substitution, the major haem degradation product was biliverdin starting from the 5 min time point. Thus, the major haem degradation product for WT and F23W MhuD is mycobilin, as previously reported for WT enzyme, ${ }^{17}$ but when the population of the planar substrate conformation is increased by the W66F substitution, biliverdin becomes the major enzyme product.

The major product of the planar conformation is $\alpha$-biliverdin 
Closer analysis of the minor product for WT enzyme revealed four isomers that could be separated by LC. The LC gradient was optimized to separate four $\mathrm{m} / \mathrm{z} 583$ species with retention times of 38.7, 39.0, 39.4, and $40.2 \mathrm{~min}$ (Fig. 5). The

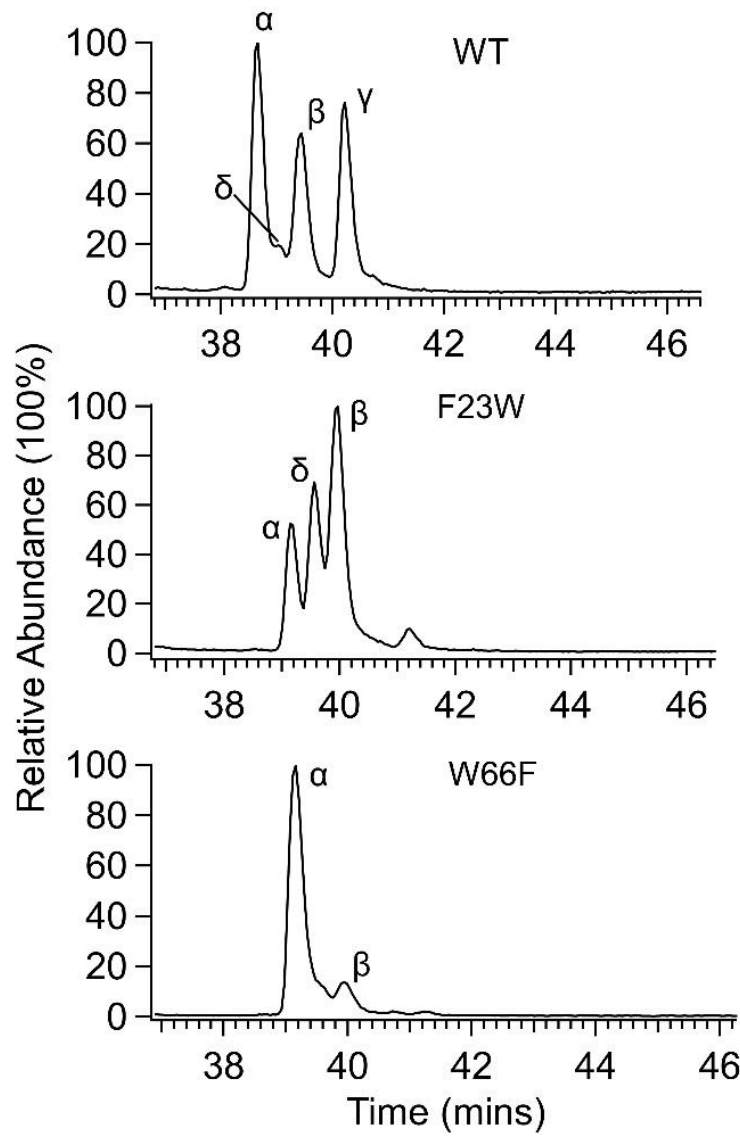

Fig. 5 Tandem LC-MS reveals that second-sphere substitutions in the MhuD active site change the isomer(s) of biliverdin produced by the enzyme. WT MhuD produces a mixture of all four biliverdin isomers, suggesting the minor product of MhuD could arise from coupled oxidation (top). F23W MhuD-catalysed haem degradation also yields a mixture of biliverdin isomers, and the minimal production of $\gamma$-biliverdin may be due to steric protection of the $\gamma$-meso carbon of porphyrin by Trp23 (middle). The major product of W66F MhuD-catalyzed haem degradation is $\alpha$-biliverdin (bottom). Biliverdin isomers were resolved by reversed-phase liquid chromatography with a $\mathrm{C}_{18}$ column and a mobile phase linear gradient of acetonitrile in water.

species with 38.7, 39.0, and 39.4 min retention times could be confidently assigned to $\alpha-, \delta$-, and $\beta$-biliverdin, respectively, based upon comparison of tandem LC-MS data to previously published data for HOs from Pseudomonas aeruginosa (Fig. S9). ${ }^{25,} 26$ The $\mathrm{m} / \mathrm{z} 583$ species with a 40.2 min retention time was also analysed by tandem LC-MS yielding a major fragment ion at $\mathrm{m} / \mathrm{z} 555$ and a minor fragment at $\mathrm{m} / \mathrm{z} 297$, which is consistent with assignment of this species to $\gamma$-biliverdin. When the ruffled conformation was favoured by introducing the F23W substitution, a mixture of $\alpha-, \delta$-, and $\beta$-biliverdin was observed. Although $\gamma$-biliverdin production by F23W MhuD was minimal, it is important to note that the distal side of the $y$-meso carbon is expected to be blocked by the Trp23 side-chain in the F23W variant. ${ }^{10}$ Altogether, these data reveal that the biliverdin generated by the ruffled haem conformation is a mixture of regioisomers, suggesting that these products may be derived from generation of a hydroxyl radical within the enzyme active site.

When the planar substrate conformation was stabilized by the W66F substitution, tandem LC-MS identified the major reaction product as $\alpha$-biliverdin. LC separation of the biliverdin isomers for W66F MhuD identified a major product with a retention time of 39.1 and a minor product with a retention time of $39.9 \mathrm{~min}$ (Fig. 5). The assignments of these species to biliverdin isomers based upon retention time alone was ambiguous, so tandem LC-MS was used to identify the two biliverdin isomers produced by W66F variant. These experiments identified the major product to be $\alpha$-biliverdin and the minor product to be $\beta$-biliverdin. It should be noted that $\alpha$ biliverdin is also the major haem degradation product for R26S MhuD. ${ }^{20}$ These observations suggest that $\alpha$-biliverdin production by R26S and W66F MhuD may be an enzymatic reaction, albeit one that proceeds at a slower rate than mycobilin production by WT enzyme. Since the W66F substitution is known to favour population of the planar substrate conformation, ${ }^{11}$ these data identify a correlation between population of the planar substrate conformation and $\alpha$-biliverdin production.

\section{The ruffled conformation promotes haem monooxygenation}

A kinetic model for haem monooxygenation was developed that accounts for the spectral differences between the WT, F23W, and W66F MhuD reaction products. Haem monooxygenation by MhuD is formally a third-order reaction dependent upon the concentrations of haem, molecular oxygen, and reductant. ${ }^{18}$ However, under the reaction conditions employed in this study, the reaction was pseudo-first order due to a molar excess of molecular oxygen and reductant. Previous studies have employed a pseudo-first order kinetic model to extract a rate constant for this rate-limiting step of haem degradation by non-canonical HOs. ${ }^{11,} 27$ However, the observations of different enzyme products for different enzyme variants reported here identifies a critical flaw in the equation used to model the data since it assumes that the products have zero absorbance at the Soret band wavelength (Fig. 2). Here, as detailed in the ESI, we have derived an equation to model pseudo-first order haem monooxygenation by WT, F23W, and W66F MhuD in a consistent manner. This will allow us to make a meaningful comparison of the haem monooxygenation rates for each enzyme variant.

Analyses of the UV/Vis Abs data within this framework revealed that increased population of the ruffled substrate conformation resulted in a faster haem monooxygenation rate. When the UV/Vis Abs data was analysed for WT enzyme, the analysis yielded a rate of $0.080 \pm 0.005 \mathrm{~min}^{-1}$ for haem monooxygenation (Fig. 6). The same analysis was applied to the UV/Vis Abs data for F23W MhuD, which favours the ruffled haem conformation, and a pseudo-first order rate constant of $0.15 \pm 0.02 \mathrm{~min}^{-1}$ was obtained. Finally, the analysis was repeated for the W66F variant, which favours the planar haem conformation, yielding a pseudo-first order rate constant of 
$0.005 \pm 0.004 \mathrm{~min}^{-1}$. These data identify a clear correlation between increased population of the ruffled haem conformation and an increased haem monooxygenation rate. Perhaps most surprisingly, the F23W variant of MhuD is a more efficient haem monooxygenase than WT enzyme! These data

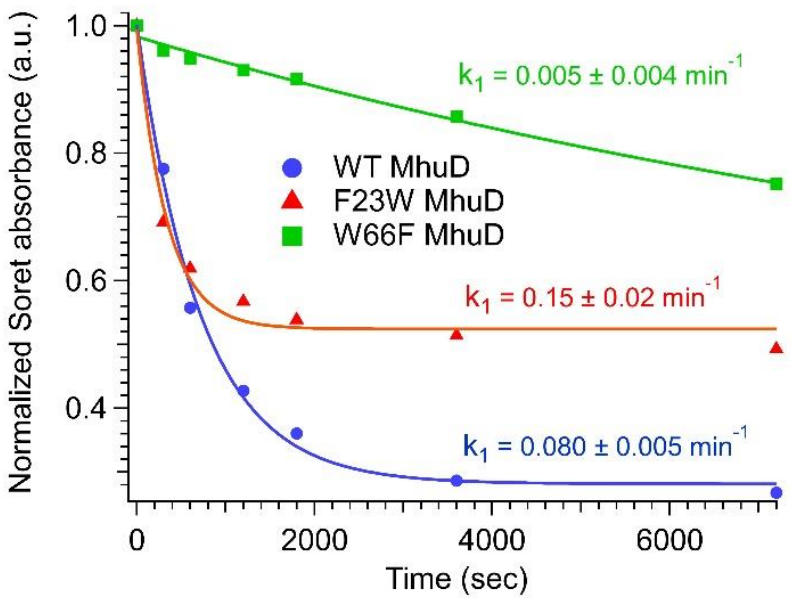

Fig. 6 The pseudo-first order rate of haem monooxygenation by MhuD is correlated with population of the ruffled haem conformation. The MhuD variant housing the most ruffled haem, F23W MhuD, has the fastest haem monooxygenation rate. The MhuD variant housing the least ruffled haem, W66F MhuD, has the slowest haem monooxygenation rate. The three curves do not reach the same absorption intensity after $2 \mathrm{~h}$ due to spectral differences between the products of these three enzyme variants. The UV/Vis Abs data depicted in Fig. 2 were fit to equation 1.

strongly suggest that access to the ruffled substrate conformation is essential for MhuD-catalysed monooxygenation of haem to meso-hydroxyhaem.

\section{The planar conformation promotes substrate dioxygenation}

A kinetic model was developed to describe the time course of the meso-hydroxyhaem intermediate created by haem monooxygenation and destroyed by dioxygenation to mycobilin. In the presence of molar excesses of molecular oxygen and reductant, hame oxygenation to mycobilin via meso-hydroxyhaem can be described by two consecutive pseudo-first order reactions. ${ }^{17}$ The initial pseudo-first order reaction forms meso-hydroxyhaem from monooxygenation of haem with a rate constant $k_{1} \cdot{ }^{18}$ This is the same haem monooxygenation reaction that was analysed above, and $k_{1}$ was constrained to the value determined by monitoring destruction of the haem Soret band. The second pseudo-first order reaction is dioxygenation of meso-hydroxyhaem to mycobilin with a rate constant of $k_{2}$. As detailed in the ESI, a kinetic model was derived for the formation and destruction of mesohydroxyhaeme where the only unknown values were $k_{2}$ and the molar extinction coefficient of MhuD-bound mesohydroxyhaem. By analysing the time-course of the $620 \mathrm{~nm}$ band observed for F23W MhuD, we will be able to estimate $k_{2}$ for this enzyme variant.

Analyses of the UV/Vis Abs data within the framework of this model revealed that increased population of the planar substrate conformation yielded a faster dioxygenation rate. In order to improve the time resolution of the data, the UV/Vis Abs assay for F23W MhuD was repeated at room temperature under otherwise similar conditions (Fig. 7). Analysis of the Soret band destruction by F23W MhuD at room temperature yielded a haem monooxygenation rate $\left(k_{1}\right)$ of $0.0253 \pm 0.0002 \mathrm{~min}^{-1}$, which is expectedly slower than the reaction carried out at 37
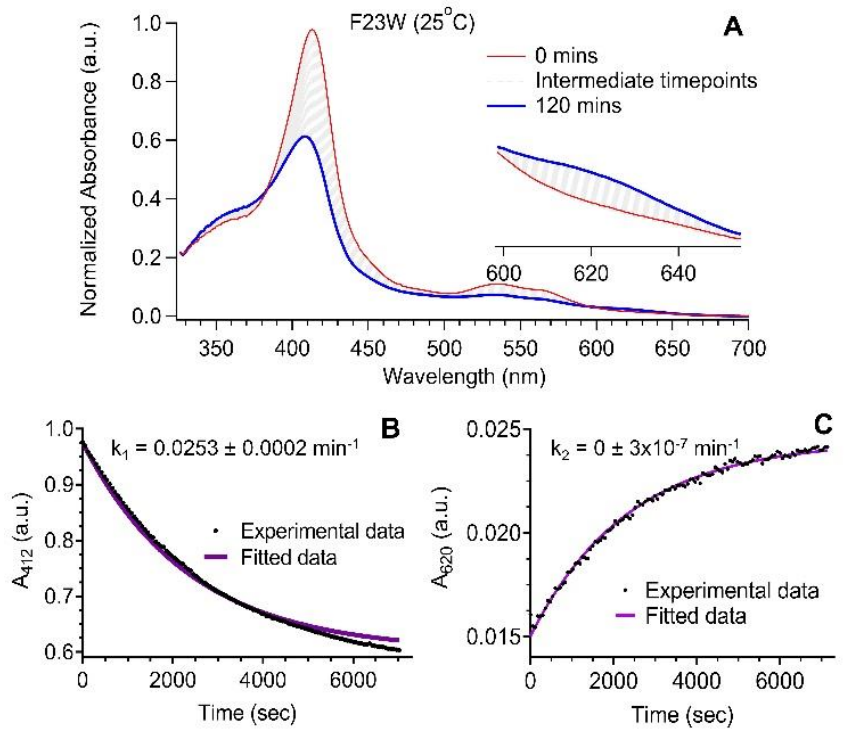

Fig. 7 F23W MhuD-catalyzed haem degradation was slowed by decreasing the temperature to $25{ }^{\circ} \mathrm{C}$ yielding improved data resolution for build-up of the mesohydroxyhaem intermediate (panel A). Kinetic analyses of the time-courses for UV/Vis absorption at $412 \mathrm{~nm}$ (panel B) and $620 \mathrm{~nm}$ (panel C) revealed that the F23W substitution changes the rate-limiting step of haem degradation by MhuD. The $412 \mathrm{~nm}$ and $620 \mathrm{~nm}$ data were fit to equations 1 and 2, respectively.

${ }^{\circ} \mathrm{C}$. Next, the formation and degradation of meso-hydroxyhaem band was satisfactorily modelled by the sequential first-order kinetic model where the rate of meso-hydroxyhaem formation was set to be equal to the rate of haem monooxygenation. By fitting the time course of the $620 \mathrm{~nm}$ Abs band to this model, we extracted a vanishingly small $k_{2}$ for F23W MhuD-catalysed meso-hydroxyhaem degradation. Since meso-hydroxyhaem formation by WT and W66F MhuD was not observed under similar conditions, it can be concluded that the F23W substitution has changed the rate-limiting step of the reaction. The F23W substitution decreases population of the planar substrate conformation, ${ }^{12}$ so there is a correlation between population of the planar substrate conformation and efficient meso-hydroxyhaem dioxygenation.

\section{Discussion}

\section{The identity of the MhuD product depends upon the substrate conformation}

The data presented in this article for F23W MhuD, a variant that stabilizes the ruffled conformation, clearly demonstrates that MhuD converts ruffled haem to mycobilin. The kinetic analysis presented above reveals that population of the ruffled conformation favours rapid haem monooxygenation since F23W MhuD has the largest $k_{1}$ (Fig. 6). Based upon the 
observation that mycobilin is the major product of F23W MhuD (Fig. 4), and the report that only the $\beta$ and $\delta$ isomers of mesohydroxyhaem are converted to mycobilin by MhuD, ${ }^{18}$ MhuDcatalysed monooxygenation of ruffled haem is regiospecific for the $\beta$ - and $\delta$-meso carbons of the porphyrin ring. However, dioxygenation by F23W MhuD is extremely slow (Fig. 7), which

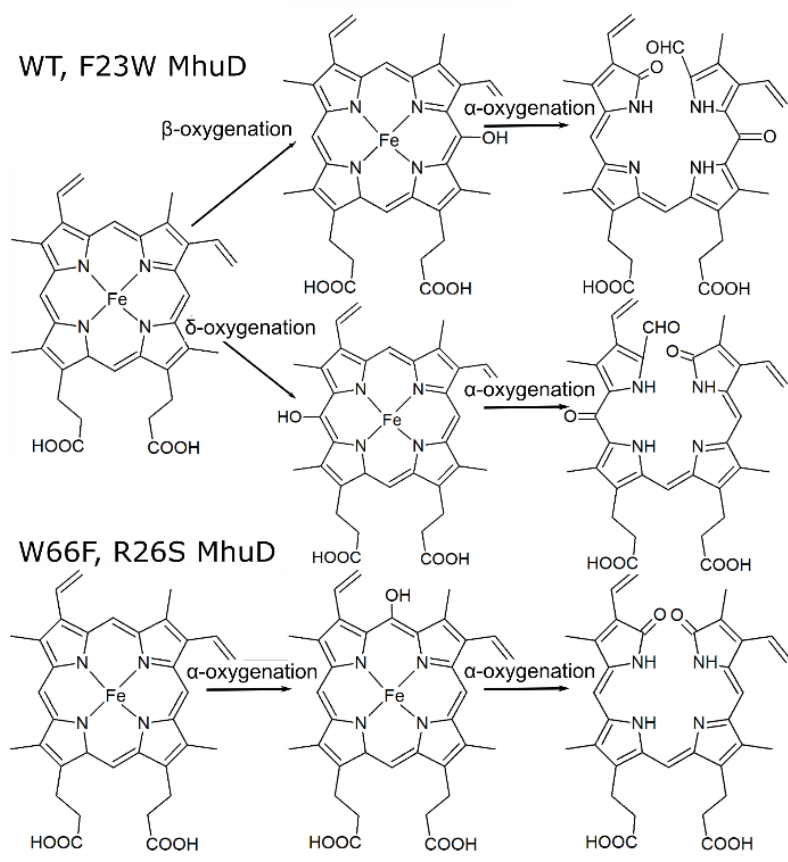

Fig. 8 The regioselectivity of MhuD-catalysed haem monooxygenation depends upon second-sphere interactions in the enzyme active site, but all characterized MhuD variants catalyse $\alpha$-oxygenation of meso-hydroxyhaem. Based upon the data presented here, WT and F23W MhuD catalyse monooxygenation of the $\beta$ - and $\delta$-meso carbons of haem en route to mycobilins. W66F MhuD catalyses regiospecific oxygenation of the $\alpha$ meso carbon, which was previously observed for R26S MhuD. ${ }^{20}$

means that MhuD cannot rapidly dioxygenate ruffled mesohydroxyhaem to mycobilin. Most likely, this is due to a rufflinginduced electronic structure change for meso-hydroxyhaem, ${ }^{17}$ whose anion can best be described as: ferric porphyrin anion ketone, ferric porphyrin enolate, or ferrous porphyrin hydroxyl radical depending upon the relative energies of the iron- and porphyrin-based orbitals. ${ }^{28,29}$ Finally, the minor products of MhuD-catalysed ruffled hame degradation, a mixture of biliverdin isomers (Fig. 5), can be attributed to coupled oxidation of haem. ${ }^{30} \mathrm{It}$ is important to once again note that the physiological redox partner for MhuD has not yet been identified, and these side products could be artefacts arising from an overpotential for ascorbate or lack of a structural change induced by the protein-protein complex. Thus, the ruffled substrate conformation supports rapid haem monooxygenation to meso-hydroxyhaem by MhuD, but this conformation alone is not sufficient for rapid oxygenation of haem to mycobilin.

On the other hand, the data presented here for the W66F variant, which favours the planar substrate conformation, reveals that MhuD degrades planar haem to $\alpha$-biliverdin. The major product of W66F MhuD-catalysed haem degradation is $\alpha$ biliverdin (Fig. 5), which is only oxygenated at the $\alpha$-meso carbon position, so W66F MhuD-catalysed haem monooxygenation must proceed through an $\alpha$-mesohydroxyhaem intermediate (Fig. 8). The rate of $\alpha$-meso carbon hydroxylation of planar haem by MhuD is at least 30 times slower than the rate of $\beta / \delta$-meso carbon hydroxylation by ruffled haem (Fig. 6), which explains why mycobilin is the major

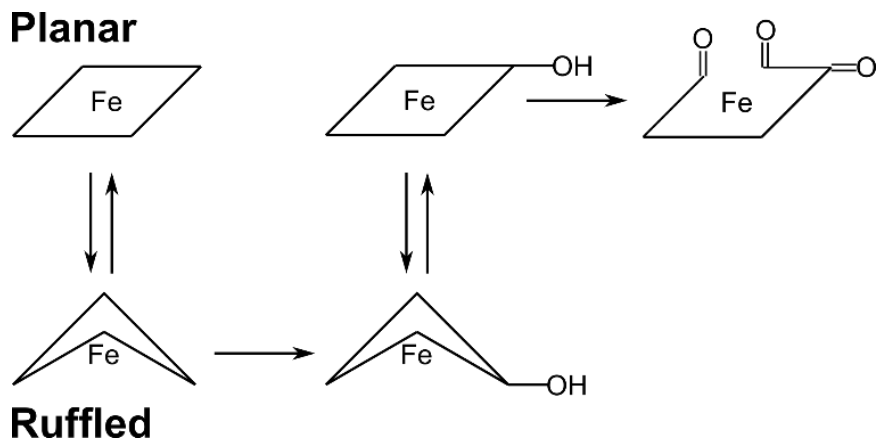

Fig. 9 Proposed mechanism for MhuD-catalysed haem oxygenation. Following haem substrate binding, an equilibrium is established between planar and ruffled haem. MhuD monooxygenates ruffled haem, and an equilibrium is established between planar and ruffled meso-hydroxyhaem. Finally, MhuD catalyses dioxygenation of planar mesohydroxyhaem.

product of WT enzyme where both conformations are accessible. ${ }^{10,12}$ The meso-hydroxyhaem intermediate was not observed for UV/Vis Abs-monitored haem degradation by WT or W66F MhuD (Fig. 2), indicating that this is not the ratelimiting step for degradation of planar haem by MhuD and $k_{2}>$ $0.080 \pm 0.005 \mathrm{~min}^{-1}$. $\alpha$-meso carbon oxygenation of planar meso-hydroxyhaem by MhuD is rapid. Finally, the observation of mycobilin as a minor product for W66F MhuD-catalysed haem degradation can be explained by limited access to the ruffled substrate conformation in this variant. The haem substrate of W66F MhuD exists in a dynamic equilibrium between planar and ruffled haem that favours planar haem, but does not fully exclude ruffled haem. ${ }^{11}$ Despite the fact that MhuD converts haem to $\alpha$-biliverdin, this substrate conformation does facilitate the second oxygenation reaction catalysed by the enzyme, namely, $\alpha$-meso carbon oxygenation of meso-hydroxyhaem.

\section{A proposed mechanism for haem oxygenation by MhuD}

The data presented in this article allows us to propose a more detailed mechanism for MhuD-catalysed haem monooxygenation than was previously available. Based upon a previous study where researchers fed synthetic mesohydroxyhaem to MhuD, the mechanistic proposal prior to this publication was that MhuD catalyses regioselective monooxygenation of haem to $\beta$ - and $\delta$-mesohydroxyhaem. ${ }^{18} \mathrm{~A}$ recent hybrid quantum mechanics / molecular mechanics (QM/MM) study attributed the regioselectivity of MhuDcatalysed haem monooxygenation to a putative hydrogen bond between Asn7 and the terminal oxygen of the putative ferrichydroperoxohaem intermediate. ${ }^{31}$ The UV/Vis Abs data presented here identifies another structural consideration for regioselective haem monooxygenation by MhuD. To be specific, the kinetic analysis of the haem monooxygenation reaction 
reveals that haem must adopt a ruffled conformation prior to monooxygenation (Fig. 9). Interestingly, the trend where $k_{1}$ increases with increasing haem ruffling cannot be explained by steric considerations because the ruffling deformation induced by the MhuD active site pushes the $\beta$ - and $\delta$-meso carbons away from a distal hydroperoxo ligand and towards the proximal His (PDB ID 4NL5). ${ }^{10}$ Instead, the monooxygenation rate enhancement is likely derived from an electronic structure change similar to that previously reported for IsdG. ${ }^{32}$ These data elucidate a mechanism where a dynamic equilibrium between planar and ruffled haem exists within the MhuD active site, ${ }^{12}$ but haem monooxygenation only proceeds for the ruffled conformation.

The data presented here also demands a revision of the proposed mechanism for meso-hydroxyhaem dioxygenation by MhuD. The same synthetic meso-hydroxyhaem study noted above also revealed that MhuD catalyses dioxygenation of the $\alpha$-meso carbon of $\beta$ - and $\delta$-meso-hydroxyhaem to mycobilin- $a$ and $-b,{ }^{18}$ and attributed the novel reactivity from canonical $\mathrm{HOs}$ to a ruffling-induced electronic structure change. ${ }^{33,} 34$ The QM/MM study noted above developed a model for this proposed reaction by predicting the energy changes associated with adding molecular oxygen to the $\alpha$-meso carbon of ruffled $\delta$-meso-hydroxyhaem. ${ }^{31}$ However, the UV/Vis Abs and MS data presented in this article indicates that meso-hydroxyhaem must adopt a planar conformation prior to further oxygenation (Fig. 9). A likely source of the discrepancy between the experimental data presented here and the computational data in the literature is that the setup of the $Q M / M M$ calculations froze the protein in the conformation observed for MhuD-haem-CN (PDB ID 4NL5), ${ }^{10}$ but at least three other protein conformations exist. ${ }^{13,14}$ The novel reactivity of the meso-hydroxyhaem form of MhuD could still be due to an electronic structure change, but this electronic structure change is not derived from substrate ruffling. Alternatively, the MhuD active site could catalyse meso-hydroxyhaem dioxygenation in a manner similar to cofactor-independent oxygenases, ${ }^{35}$ with Streptomyces coelicolor ActVA-Orf6 being a particularly relevant precedent due to the structural homology of MhuD and ActVA-Orf6. ${ }^{13,} 36,37$ Thus, although this study provides significant new insight into the mechanism of meso-hydroxyhaem dioxygenation by MhuD, several important open questions remain.

\section{MhuD relies upon a dynamic, out-of-plane, substrate distortion}

Perhaps the single most important contribution of the data presented in this article to our understanding of the MhuD mechanism is that enzymatic turnover requires a dynamic substrate. The haem monooxygenation reaction catalysed by MhuD requires access to the ruffled substrate conformation. This conformation is derived from a pseudo Jahn-Teller distortion that mixes ${ }^{2} A_{2 u}$ character into the ${ }^{2} B_{2 g}$ ground state yielding partial radical character on the porphyrin meso carbons and promoting their combination with a hydroxyl radical generated in situ (Fig. 10).12, 15, 16 However, this correlation between haem out-of-plane ruffling and electronic structure is specific for low-spin ferric haem. Meso-hydroxyhaem can be described as a ferrous oxyhaem with a porphyrin-based radical. ${ }^{28,} 29$ The pseudo Jahn-Teller vibronic mixing described above lowers the energy of the ${ }^{2} B_{2 g}$ state at the expense of raising the energy of the ${ }^{2} A_{2 u}$ state. This means that ruffled meso-hydroxyhaem is likely to have ${ }^{2} A_{1 u}$ character with the radical localized to the porphyrin pyrrole carbons, the planar meso-hydroxyhaem is likely to have ${ }^{2} A_{2 u}$ character with the radical localized to the porphyrin meso carbons, and meso-hydroxyhaem oxygenation is most favourable for a planar substrate. ${ }^{38,} 39$ In essence, the bifunctionality of MhuD requires

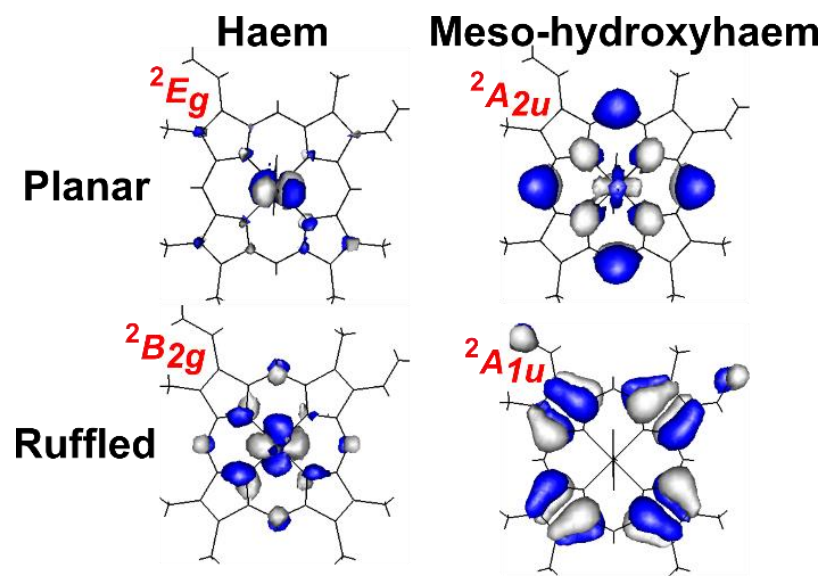

Fig. 10 Expected singly-occupied molecular orbitals for planar and ruffled haem and meso-hydroxyhaem, and the corresponding electronic states. To place spin density on the haem meso carbons, haem must adopt a ruffled conformation. ${ }^{11}$ Conversely, mesohydroxyhaem must adopt a planar conformation to place spin density on the meso carbons. Note, the ${ }^{2} E_{g}$ state is doubly-degenerate and only one of the $e_{g}$ orbitals is shown.

access to two substrate conformations because these conformations differentially tune the electronic structures of the haem substrate and the meso-hydroxyhaem intermediate.

The data presented in this article have revealed that haem dynamics are an essential consideration for MhuD-catalysed haem degradation, and further consideration for the role of haem dynamics in all haem-dependent proteins is warranted. Ever since the types of haem out-of-plane distortion were categorized over 20 years ago, ${ }^{8}$ correlations between out-ofplane haem distortion and protein function have been regularly identified.9, 40 However, what has not been as generally appreciated is that the out-of-plane vibrational modes are on the order of thermal energy at $37{ }^{\circ} \mathrm{C}\left(220 \mathrm{~cm}^{-1}\right) .41,42$ For MhuD, this means that the haem substrate of WT enzyme is regularly interconverting between the planar and ruffled substrate conformations. ${ }^{12}$ In general, the energy required to trigger an out-of-plane haem distortion means that we need to envision protein-bound haem as continually undergoing dynamic out-ofplane distortion from the equilibrium geometry captured by $\mathrm{X}$ ray crystallography. As dramatically exemplified by haem oxygenases, ${ }^{10,43}$ these structural dynamics are tied to electron dynamics that significantly influence haem reactivity. Haem has a dense cluster of iron- and porphyrin-based orbitals near the HOMO/LUMO gap, ${ }^{39}$ and subtle structural changes can alter the orbital occupations resulting in dramatic changes to haem reactivity. Thus, haem cannot be considered a classic organic substrate where static structure determines function, and the influence of haem dynamics on haem electronic structure represents an exciting open challenge for physical bioinorganic chemistry. 


\section{Conclusions}

In conclusion, this article presents new data which reveals that a dynamic haem substrate is critical for MhuD-catalysed haem degradation. UV/Vis Abs and MS data for MhuD variants that favour either a ruffled or planar substrate conformation have shown that the MhuD product depends upon the substrate conformation. Single-turnover kinetic analyses of the UV/Vis Abs data have demonstrated that the rates of haem monooxygenation and meso-hydroxyhaem dioxygenation also depend upon the substrate conformation. These observations have enabled us to prepare a revised enzymatic mechanism that is consistent with both previously published data and the new data presented here. This mechanism relies upon a dynamic haem substrate and raises the question of whether haem dynamics should be more carefully considered as a significant contributor to haem function.

\section{Conflicts of interest}

There are no conflicts to declare.

\section{Acknowledgements}

The authors acknowledge Celia Goulding (University of California-Irvine) for helpful discussions and a critical reading of the manuscript. M.D.L. thanks the National Institutes of Health (R01-GM114277) for financial support.

\section{References}

1 J. T. Jarrett, S. Huang and R. G. Matthews, Biochemistry, 1998, 37, 5372-5382.

2 V. Bandarian, M. L. Ludwig and R. G. Matthews, Proc. Natl. Acad. Sci., 2003, 100, 8156-8163.

3 J. C. Evans, D. P. Huddler, M. T. Hilgers, G. Romanchuk, R. G. Matthews and M. L. Ludwig, Proc. Natl. Acad. Sci., 2004, 101, 37293736.

4 J. Monod, J. Wyman and J.-P. Changeux, J. Mol. Biol., 1965, 12, 88118.

5 M. F. Perutz, Nature, 1970, 228, 726-734.

6 Y. Yuan, M. F. Tam, V. Simplaceanu and C. Ho, Chem. Rev., 2015, 115, 1702-1724.

7 W. Jentzen, M. C. Simpson, J. D. Hobbs, X. Song, T. Ema, N. Y. Nelson, C. J. Medforth, K. M. Smith, M. Veyrat, M. Mazzanti, R. Ramasseul, J.-C. Marchon, T. Takeuchi, W. A. Goddard and J. A. Shelnutt, J. Am. Chem. Soc., 1995, 117, 11085-11097.

8 W. Jentzen, X.-Z. Song and J. A. Shelnutt, J. Phys. Chem. B, 1997, 101, 1684-1699.

9 J. A. Shelnutt, X.-Z. Song, J.-G. Ma, S.-L. Jia, W. Jentzen and C. J. Medforth, Chem. Soc. Rev., 1998, 27, 31-41.

10 A. B. Graves, R. P. Morse, A. Chao, A. Iniguez, C. W. Goulding and M. D. Liptak, Inorg. Chem., 2014, 53, 5931-5940.

11 A. B. Graves, M. T. Graves and M. D. Liptak, J. Phys. Chem. B, 2016, 120, 3844-3853.

12 A. B. Graves, E. H. Horak and M. D. Liptak, Dalton Trans., 2016, 10058-10067.

13 N. Chim, A. Iniguez, T. Q. Nguyen and C. W. Goulding, J. Mol. Biol., 2010, 395, 595-608.
14 A. Chao, K. H. Burley, P. J. Sieminski, R. de Miranda, X. Chen, D. L. Mobley and C. W. Goulding, Biochemistry, 2019, 58, 4610-4620.

15 P. Garcia-Fernandez, I. B. Bersuker and J. E. Boggs, J. Chem. Phys., 2006, 125, 104102.

16 I. B. Bersuker, Chem. Rev., 2013, 113, 1351-1390.

17 S. M. Nambu, T.; Goulding, C.W.; Takahashi, S.; Ikeda-Saito, M., J. Biol. Chem., 2013, 288, 10101-10109.

18 T. Matsui, S. Nambu, C. W. Goulding, S. Takahashi, H. Fujii and M. Ikeda-Saito, Proc. Natl. Acad. Sci., 2016, 113, 3779-3784.

19 T. Matsui, M. Unno and M. Ikeda-Saito, Acc. Chem. Res., 2010, 43, 240-247.

20 A. Chao and C. W. Goulding, Biochemistry, 2019, 58, 489-492.

21 B. Thakuri, A. B. Graves, A. Chao, S. L. Johansen, C. W. Goulding and M. D. Liptak, Metallomics, 2018, 10, 1560-1563.

22 B. Bothner, R. Chavez, J. Wei, C. Strupp, Q. Phung, A. Schneemann and G. Siuzdak, J. Biol. Chem., 2000, 275, 1345513459.

23 E. A. Berry and B. L. Trumpower, Anal. Biochem., 1987, 161, 1-15. 24 A. Wilks and P. R. Ortiz de Montellano, J. Biol. Chem., 1993, 268, 2235722362.

25 K. D. Barker, K. Barkovits and A. Wilks, J. Biol. Chem., 2012, 287, 18342-18350.

26 M. J. O'Neill and A. Wilks, ACS Chem. Biol., 2013, 8, 1794-1802.

27 G. Ukpabi, S. J. Takayama, A. G. Mauk and M. E. Murphy, J. Biol. Chem., 2012, 287, 34179-34188.

28 K. M. Matera, S. Takahashi, H. Fujii, H. Zhou, K. Ishikawa, T. Yoshimura, D. L. Rousseau, T. Yoshida and M. Ikeda-Saito, J. Biol. Chem., 1996, 271, 6618-6624.

29 Y. Liu, P. Moenne-Loccoz, T. M. Loehr and P. R. Ortiz de Montellano, J. Biol. Chem., 1997, 272, 6909-6917.

30 J. W. Legge and R. Lemberg, Biochem. J., 1941, 35, 353-362.

31 C. Yuan, Y. Zhang, H. Tan, X. Li, G. Chen and Z. Jia, Phys. Chem. Chem. Phys., 2020, 22, 8817-8826.

32 M. A. Conger, A. R. Cornetta and M. D. Liptak, Inorg. Chem., 2019, 58, 15455-15465.

33 I. Morishima, H. Fujii, Y. Shiro and S. Sano, Inorg. Chem., 1995, 34, 1528-1535.

34 X. Zhang, H. Fujii, K. Mansfield Matera, C. T. Migita, D. Sun, M. Sato, M. Ikeda-Saito and T. Yoshida, Biochemistry, 2003, 42, 74187426.

35 S. Fetzner and R. A. Steiner, Appl. Microbiol. Biotechnol., 2010, 86, 791-804.

36 G. Sciara, S. G. Kendrew, A. E. Miele, N. G. Marsh, L. Federici, F. Malatesta, G. Schimperna, C. Savino and B. Vallone, EMBO J., 2003, 22, 205-215.

37 E. P. Skaar, A. H. Gaspar and O. Schneewind, J. Biol. Chem., 2004, 279, 436-443.

38 M. Gouterman, J. Chem. Phys., 1959, 30, 1139-1161.

39 F. A. Walker, Coord. Chem. Rev., 1999, 185-186, 471-534.

40 M. O. Senge, S. A. MacGowan and J. M. O'Brien, Chem. Commun., 2015, 51, 17031-17063.

41 M. Kubo, F. Gruia, A. Benabbas, A. Barabanschikov, W. R. Montfort, E. M. Maes and C. P.M., J. Am. Chem. Soc., 2008, 130, 9800-9811.

42 V. Karunakaran, Y. Sun, A. Benabbas and P. M. Champion, J. Phys. Chem. B, 2014, 118.

43 S. J. Takayama, G. Ukpabi, M. E. Murphy and A. G. Mauk, Proc. Natl. Acad. Sci., 2011, 108, 13071-13076. 